\title{
Über die Darstellung der Fuchs'schen Functionen erster Familie durch unendliche Producte.
}

\author{
Von Otto Biermann in Klagenfurt.
}

Will man die Theorie der Fuehs'schen Functionen derart entwickeln, dass man solche Functionen bei vorgegebenen Nullund Unendlichkeitsstellen bilden kann, reichen die $\Theta$. F uchsschen Functionen *) nicht aus, weil man deren Nullstellen nicht anziugeben weiß. Man bedarf vielmehr Hilfsfunctionen, bei denen die Null- und Unendlichkeitsstellen stets ersichtlich bleiben, also Producte gewisser Primfunctionen. ${ }^{* *}$ )

Ich stelle in folgendem zunächst diejenigen Sätże kurz zusammen, welche für die Bildung einer Gruppe linearer Substitutionen erster Familie nöthig sind, um nicht allein Gelegenheit zu haben, späterhin gebrauchte Bezeichnungen einzuführen, sondern um auch eine bestimmte Form der den Einheitskreis nicht ändernden linearen Substitutionen abzuleiten und bei einem allgemeinen Polygon von $2 n$ Seiten und beliebigem Range eine Conjunction der Seiten anzugeben. Im zweiten Paragraphen construiere ich die einfachste Form éiner eindeutigen, analytischen Function, welche an einer Stelle $a$ des Fundamentalpolygons und ferner an den durch die Substitutionen $\left(z, f_{r}(z)\right)$ einer Gruppe erster Familie transformierten Stellen $a_{r}=f_{r}(a)$ von der ersten Ordnung verschwindet, indes jede Stelle des durch die Substitutionen nicht alterierten Einheitskreises eine wesentlich singuläre Stelle ist.

Der Kernpunkt der.Untersuchung liegt in dem Nachweise, dass dann, wenn $b_{r}$ die gegenüber dem Fundamentkreise zu $a_{r}$ symmetrische Stelle ist, die Reihe

$$
\underset{v}{\mathbf{a}}\left|a_{\nu}-b_{\nu}\right|^{2}
$$

convergiert. Auf den auffallenden Umstand, dass hier der

*) Poincaré, Acta mathematica. Bd. 1 .

**) B i e rma n n, Sitzber. d. Akad. d.W. in Wien. Bd. 92. Novemiberheft 1885.

Monatsh. f. Mathematik n. Physik. 1. Jahrg., 2. Heft. 
Exponent 2 auftritt, indes dann, wenn $a_{r}$ die Nullstellen der Weierstrab'schen Function $\sigma(z)$ bedeuten, die Reihe

$$
\underset{\nu}{\mathbf{s}^{\prime}}\left|a_{\nu}-\infty\right|^{2}=\mathbf{\Sigma}^{\prime}\left|\frac{1}{a_{\nu}}\right|^{2}
$$

divergiert, wende ich meine Aufmerksamkeit.

Ferner untersuche ich das Verhalten der besagten Function gegenüber einer Substitution der Gruppe, leite dann den allgemeinsten Ausdruck einer innerhalb des Einheitskreises eindeutigen analytischen Function mit vorgegebenen Null- und Unendlichkeitsstellen ab, und kennzeichne auch deren Verhalten gegenüber einer Substitution. Dann ist es möglich, die Bedingungen anzugeben; unter welchen die Function eine $\mathrm{F}$ u c hs'sche wird; doch habe ich dieselben noch nicht anf die nothwendigen und hinreichenden zurückgeführt.

\section{\$. 1 ,}

Es sei in der durch die reelle Achse begrenzten positiven Halbebene oder innerhalb des Einheitskreises der Ebene einer unbeschränkten Veränderlichen $\approx$ eine Eintheilung in convexe Polygone vollzogen, deren Begrenzungen durch Kreisbogen gegeben sind, welche die reelle Achse oder den Einheitskreis senkrecht schneiden, und es bestehe eine Gruppe linearer Substitutionen:

$$
\left(z, \frac{\alpha_{\nu} z+\beta_{\gamma}}{\gamma_{\nu} z+\delta_{\nu}}\right) \text { oder }\left(z, f_{\nu}(z)\right)
$$

welche die reelle Achse, beziehungsweise den „Fundamentalkreis" nicht alterieren und die genannten Polygone ineinander überführen,

Um einen bestimmten Fall vor uns zu haben, nehmen wir ein für allemal an, dass die begrenzenden Seiten der Polygone niemals einen Theil der reellen Achse oder des Einheitskreises ausmachen und die Ecken stets innerhalb des durch die Eintheilung ïberdeckten Bereiches der $z$ Ebene liegen. Die Seiten seien also von der "ersten Art" und die Ecken von der "ersten Kategorie".

Zwei Stellen heißen äquivalent oder entsprechend und zwei Figuren heißen congruent, wenn die eine die durch eine Substitution der Gruppe Transformierte der anderen ist. Die Polygone sind daher congruent. Grenzt an ein Polygon $R_{0}$ längs seiner Seite $l_{p}$ das Polygon $R_{p}$, welches durch die Substitution $\left(z, f_{p}(z)\right)$ aus $R_{0}$ hervorgeht, so werden die auf $l_{p}$ liegenden und zu $R_{p}$ gezählten Punkte durch die Substitution $\left(z, f_{p}^{-1}(z)\right)$ auf eine Seite $l_{p}^{\prime}$ von $R_{0}$ übertragen, die $R_{0}$ von dem durch Anwendung der letzten Substitution aus $R_{0}$ hervorgehenden Bereiche $R_{p}^{\prime}$ trennt. Zwei Seiten $l_{p}$ und $l_{p}^{\prime}$ der ge- 
nannten Art heißen conjugiert; sie sind congruent und müssen in dem Polygon in gerader Zahl vorkommen.

Die Ecken des Polygons lassen sich in Gruppen von ein oder mehreren einander entsprechenden Punkten bringen. Die Gesammtheit einander äquivalenter Ecken bildet einen "Cyclus". Bei der getroffenen Annahme ïber die Lage der Ecken und Seiten innerhalb des eingetheilten Bereiches gibt es nur ,geschlossene Cyclen", und die Polygone sind von der ersten Familie.

Ist $A_{1}$ eine beliebige Ecke, $B_{1}$ eine der angrenzenden Seiten, $C_{1}$ ihre conjugierte Seite, $A_{2}$ die folgende Ecke, dann wieder $B_{2}$ die nächste Seite, $C_{2}$ ihre conjugierte und $A_{3}$ die folgende Ecke u. s. f., so kommt man zu $A_{1}$ zurück und hat alle Ecken desjenigen Cyclus berührt, welchem $A_{1}$, sowie jede der besagten Ecken angehört.

Beschreibt man um $A_{1}$ einen beliebig kleinen Kreis und tritt aus dem Polygon $R_{0}$ durch die Seite $B_{1}$ in den Bereich $R_{1}$, so ist $B_{1}$, als Seite von $R_{1}$ betrachtet, homolog $C_{1}$ und die Ecke $A_{1}$ homolog $A_{2}$. Bei dem weiteren Umkreisen tritt man durch eine $B_{2}$ homologe Seite in den Bereich $R_{2}$. In diesem ist die eben überschrittene Seite homolog $C_{2}$ und die Ecke $A_{1}$ homolog $A$. So fortschreitend gelangt man endlich wieder zu dem Bereich $R_{0}$. Ähnliches gilt bei dem Umkreisen der übrigen Ecken $A_{2}, A_{3}, \ldots A_{m}$ des die Euke $A_{1}$ umfassenden Cyclus. Da die entsprechenden Winkel in congruenten Polygonen gleich groß sind. indem die linearen Substitutionen die Winkel nicht ändern, ist die Anzahl der in jeder Ecke eines $m$ gliederigen Cyclus zusammenstoßenden Bereiche dieselbe, und zwar ein ganzes Vielfache von $m$ z. B. $p m$. Die Summe der Polygonwinkel an den Ecken des $m$ gliederigen Cyclus ist dann der $p^{\text {te }}$ Theil von $2 z$.

Diese Bedingung und die frühere, über die Congruenz conjugierter Seiten sind nothwendig und hinreichend dafïr, dass zu der getroffenen Eintheilung eine discontinuierliche Gruppe linearer Substitutionen gehört. Die Gruppe ist aus denjenigen Substitutionen abzuleiten, welche die conjugierten Seiten in einander übertragen, doch gibt es zwischen diesen ,Fundamentalsubstitutionen" noch Beziehungen von der Anzahl der Cyclen, welche ausdrücken, dass man bei Umkreisung je einer Ecke des einzelnen Cyclus durch die Substitutionen zu dem Ausgangspolygon zuriuckgelangt.

Besitzt das Polygon $2 n$ Seiten und $q$ Cyclen, dann ist auf der aus dem Polygon durch Aneinanderheften seiner conjugierten Seiten und Zusammenfügen der Ecken jedes gesonderten Cyclus zu bildenden Fläche die gröbte Zahl der geschlossenen Curven, welche keine Theilung der Fläche hervor- 
rufen: $\varrho=\frac{n+1}{2}-q$. Da $\varrho$, der "Rang“. der Fläche, des Polygons und der zugehörigen Gruppe eine positive ganze Zahl sein muss, ist die Zahl $q$ der Cyclen höchstens $n+1$, und $q$ ist ungerade, wenn $n$ gerade ist, und gerade im entgegengesetzten Falle.

Umfasst der erste, zweite u. s. w., endlich der $q^{\text {te }}$ Cyclus $n_{1}, n_{2}, \ldots n_{q}$ Ecken, so ist

$$
n_{1}+n_{2}+\ldots+n_{q}=2 n \text {. }
$$

Die Anzahl der möglichen Cyclen bei einem Polygon von $2 n$ Seiten, wo $n=2 m+1$ oder $n=2 m$ ist, ist durch die Summe der Anzahl ganzzahliger Lösungen jeder der $(m+1)$ Gleichungen :

$$
x_{1}+x_{2}+\ldots+x_{n+1-\nu}=2 n(\nu=0,2,4, \ldots 2 m),
$$

gegeben, wobei $x_{1}>0, x_{\lambda} \leq x_{\lambda+1}, x_{n+1-v} \leqq n+\nu$ ist. Der Rang ist bei $n+1-v$ Cyclen $\frac{v}{2}$.

Die Anzahl der verschiedenen Cyclen bei gleich bleibendem Range ist nicht von Belang; denn die Polygone von gleicher Seitenzahl und gleichem Range lassen sich dadurch aufeinander zurückführen, dass man zu einem Polygon $R_{0}$ mit bestimmter Zuordnung seiner Seiten einen Bereich passend hinzufügt und den zufolge einer Substitution der Gruppe congruenten Bereich wegnimmt.

Theilt man z. B. $R_{0}$ durch einen Kreis, der die Begrenzung des eingetheilten Bereiches der $z$ Ebene senkrecht schneidet, in zwei Bereiche $P_{0}$ und $Q_{n}$ derart, dass zwei conjugierte Seiten $B_{i}$ und $C_{i}$ getrennt werden und stößt an die Seite $C_{i}$ von $Q_{0}$ der Bereich $R_{i}^{\prime}$, der durch einen dem früheren congruenten Bogen in $P_{i}^{\prime}$ and $Q_{i}^{\prime}$ getheilt wird, so kann man $Q_{0}+P_{i}^{\prime}$ als den für die Eintheilung fundamentalen Bereich auffassen. Doch bei diesem können die aufeinanderfolgenden Seiten in geänderter Zuordnung vorkommen, und wenngleich die Zahl der Cyclen erhalten bleibt, brauchen die einzelnen Cyclen nicht gleichviele Ecken, wie vordem in $R_{0}$ zu umfassen.

Nach diesen Bemerkungen genügt es, eine Art der Conjunction der Seiten des Polygons vom Range $\varrho$ anzugeben.

Nehmen wir an, dass unter den $n-2 \varrho+1$ Cyclen $n-2 \varrho=i$ aus einer Ecke allein bestehen und der letzte Cyclus die übrigen $2 n-i$ Ecken umfasst, welcher Fall der Gleichung

$$
1+1+\ldots+1+(2 n-i)=2 n
$$

entspricht. Schreibt man die conjugierten Seiten nebeneinander, eine erste und ihre conjugierte, die zweite und die conjugierte, u. s. w. Dann ist das Schema für die Zuordnung:

$$
\left(\begin{array}{l}
1, n+i+1 ; 2, n+i+2 ; \quad \ldots n-i, 2 n ; \\
n-i+1, n-i+2 ; \ldots n+i-1, n+i
\end{array}\right)
$$


Functionen erster Familie durch unendliche Producte.

Dieses Beispiel ist durch die folgende Figur beschrieben, wo die Ecken des mehrghiederigen Cyclus mit unteren, die übrigen mit oberen Indices versehen sind und die Seiten eine passende Bezeichnung finden. $B_{n-i}$

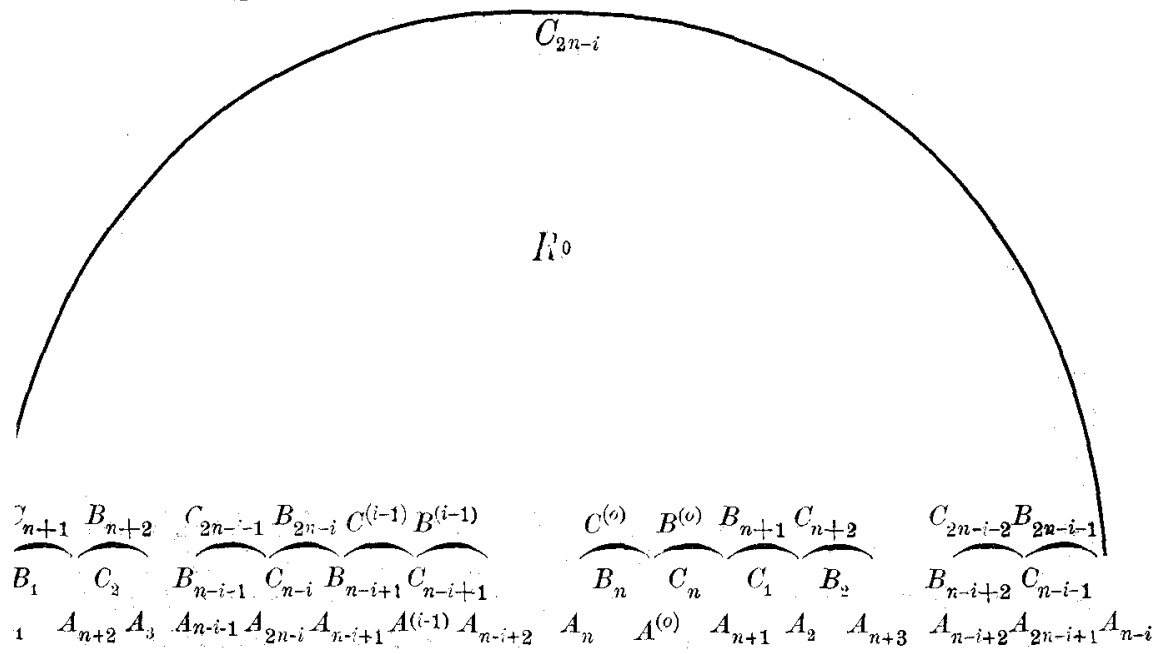

Es ist

$$
\begin{gathered}
B_{v}=C_{n+v}, C_{\nu}=B_{n+v}(\nu=1,2, \ldots n-\imath) \\
B_{n-i+j}=C^{(i-j)}, C_{n-i+j}=B^{(i-j)}(j=1,2, \ldots i)
\end{gathered}
$$

Die Summe der Winkel an den Ecken $A$ des $(2 n-i)$ gliederigen Cyclus sei $\frac{2 \pi}{p}$, der Winkel an der Ecke $A^{(j-1)}$ sei $\frac{2 \pi}{p^{(i-1)}}$.

Heißt die Substitution, welche das Polygon $R_{v}$ bei dem Austritt durch $B_{v}$ in den Bereich $R_{v}$ überführt, $\left(z, f_{v}(z)\right)$, und bezeichnet man

dann wird

$$
\begin{gathered}
f_{1} f_{2} \cdots f_{2 n-i}(z)=F(z), \\
z=F^{p}(z) .
\end{gathered}
$$

Nennt man die Substitution, welche $R_{v}$ in den an die Seite $B^{(j-1)}$ grenzenden Bereich führt, $\left(z, \varphi_{j_{-1}}(z)\right)$, wird auch

Weil aber

$$
z=\varphi_{j-1}^{p_{j-1}}(z)
$$

und

$$
\varphi_{j-1}(z)=f_{n-j+1}^{-1}(z) \quad(j=1,2, \cdots i)
$$

$$
f_{n+1}^{\prime}(z)=f_{1}^{-1}(z), f_{n+2}(z)=f_{2}^{-}(z), \ldots f_{n+(n-i)}(z) \equiv f_{n-i}^{-1}(z)
$$

ist. kann man

$$
f_{1}, f_{2}, \ldots f_{n-i}, \varphi_{v}, \varphi_{1}, \ldots \varphi_{i-1}
$$


als Fundamentalsubstitutionen betrachten und dann geht die "Fundamentalrelation" (1) in die folgende Beziehung über:

$$
\left.z={ }_{1}^{\prime} f_{1} f_{2} \cdots f_{n-i} \varphi_{i-1}^{-1} \varphi_{i-2}^{-1} \cdots \varphi_{0}^{-1} f_{1}^{-1} f_{2}^{-1} \cdots f_{n-1}^{-1}(z)\right\}^{p} \text {. }
$$

Im Falle eines Vierecks mit einem Cyclus und dem Range $\varrho=1$ hat man eine erste Seite der dritten, die zweite der vierten zuzuordnen. Sind die zugehörigen Substitutionen $\left(z, f_{1}(z)\right.$ ), $\left(z, f_{2}(z)\right)$, so lautet die Fundamentalrelation zwischen diesen:

$$
z \equiv\left(f_{1} f_{2} f_{1}^{-1} f_{2}^{-1}(z)\right)^{p} \text {. }
$$

Es handelt sich nun um die wirkliche Berechnung der Gruppen und zunächst um die Bestimmung der reellen Substitutionen

$$
t=\frac{A z+B}{C z+D}
$$

welche die reelle Achse der $z$ Ebene ungeändert lassen. Nennt man die $z$ Werte an den Ecken einer Seite des (der fraglichen Gruppe zugehörigen) Polygons $R_{0} \quad \alpha=\alpha_{1}+i \alpha_{2}, \beta=\beta_{1}+i \beta_{2}$, und die $z$ Werte an den Ecken der conjugierten Seite $\gamma=\gamma_{1}+i \gamma_{2}, \delta=\delta_{1}+i \delta_{2}$, sind weiter die conjugiert imaginären Stellen mit $\alpha^{\prime}, \beta^{\prime}, \gamma^{\prime}, \delta^{\prime}$ bezeichnet, so hat man die Coefficienten $A, B, C, D$ derart zu bestimmen, dass den Werten $z=\alpha, \beta, \alpha^{\prime}, \beta^{\prime}$ die Werte $t=\gamma, \delta, \gamma^{\prime}, \delta^{\prime}$ entsprechen. Es sollen demnach die Gleichungen bestehen

$$
\begin{aligned}
& A \beta+B-C \beta \delta-D \delta=0 \\
& A \beta^{\prime}+B-C \beta^{\prime} \delta^{\prime}-D \delta^{\prime}=0 \\
& A \alpha+B-C \alpha \gamma-D \gamma=0 \\
& A \alpha^{\prime}+B-C \alpha^{\prime} \gamma^{\prime}-D \gamma^{\prime}=0 .
\end{aligned}
$$

Die Determinante des Gleichungssystems muss verschwinden, doch diese Forderung ist erfüllt, indem die selbstverständliche Beziehung zwischen vier $z$ Werten $z_{1}, z_{2}, z_{3}, z_{4}$ und ihren entsprechenden $t$ Werten $t_{1}, t_{2}, t_{3}, t_{4}$ :

im besonderen die Gleichung

$$
\frac{t_{1}-t_{2}}{t_{1}-t_{3}}: \frac{t_{4}-t_{2}}{t_{4}-t_{3}}=\frac{z_{1}-z_{2}}{z_{1}-z_{3}}: \frac{z_{4}-z_{3}}{z_{4}-z_{3}}
$$

$$
\frac{\gamma^{\prime}-\delta}{\gamma^{\prime}-\gamma} \cdot \frac{\delta^{\prime}-\gamma}{\delta^{\prime}-\delta}=\frac{\alpha^{\prime}-\beta}{a^{\prime}-\alpha} \frac{\beta^{\prime}-\alpha}{\beta^{\prime}-\beta}
$$

nach sich zieht. Die reellen Substitutionscoefficienten werden*):

$$
\begin{aligned}
& A=\left|\begin{array}{lll}
\alpha_{1} \gamma_{1}-\alpha_{2} \gamma_{2}, & \gamma_{1}, & 1 \\
\beta_{1} \delta_{1}-\beta_{2} \delta_{2}, & \delta_{1}, & 1 \\
\beta_{1} \delta_{2}+\beta_{2} \delta_{1}, & \delta_{2}, & 0
\end{array}\right|, \quad B=\left|\begin{array}{lll}
\alpha_{1} \gamma_{1}-\alpha_{2} \gamma_{2}, & \alpha_{1}, & \gamma_{1} \\
\beta_{1} \delta_{1}-\beta_{2} \delta_{2}, & \beta_{1}, & \delta_{1} \\
\beta_{1} \delta_{2}+\beta_{2} \delta_{1}, & \beta_{2}, & \delta_{2}
\end{array}\right| \\
& D=\left|\begin{array}{lll}
\alpha_{1} \gamma_{1}-\alpha_{2} \gamma_{2}, & \alpha_{1} & 1 \\
\beta_{1} \delta_{1}-\beta_{2} \delta_{2}, & \beta_{1} & 1 \\
\beta_{1} \delta_{2}+\beta_{2} \delta_{1}, & \beta_{2} & 0
\end{array}\right|, \quad C=\left|\begin{array}{ll}
\alpha_{1}, \gamma_{1}, & 1
\end{array}\right| \begin{array}{lll}
\beta_{1}, & \delta_{1}, & 1 \\
\beta_{2}, & \delta_{2}, & 0
\end{array} \mid
\end{aligned}
$$

*) In der Arbeit von M. Poincaré lautet das dritte Glied der zweiten Horizontalreihe in der. Determinante $B \gamma_{2}$ statt $\delta_{1}$. 
Hat man auf solche Weise die reellen Fundamentalsubstitutionen gefunden, so sind deren Coefficienten noch den Bedingungen zu unterwerfen, welche die Convexität des Polygons mit sich bringt, ferner den Forderungen, welche der Satz uiber die Summe der Winkel an den Ecken jedes Cyclus verlangt und endlich den durch die Fundamentalrelationen vorgeschriebenen Beziehungen. Dann erst ist die Gruppe bestimmt.

Um auch eine Gruppe linearer Substitutionen anzugeben, welche den Einheitskreis ungeändert lassen, brauchen wir die obige Gruppe nur passend umzuformen.

Setzt man

$$
t=u+i v=\frac{a z+b}{c z+d}
$$

und verlangt, dass zufolge dieser Substitution der reellen Achse der $z$ Ebene in der $t$ Ebene der Einheitskreis entspricht, müssen die complexen Coefficienten

$$
a=a_{1}+i a_{2}, \quad b=b_{2}+i b_{2}, \quad c=c_{1}+i c_{2}, \quad d=d_{1}+i d_{2}
$$

den Gleichungen gemäß gewählt werden:

$$
\begin{aligned}
& a_{1} d_{2}-a_{2} d_{1}+c_{1} b_{2}-c_{2} b_{1}=0 \\
& a_{1} d_{1}+a_{2} d_{2}-c_{1} b_{1}-c_{2} b_{2}=0 \\
& a_{1} b_{2}-a_{2} b_{1}+c_{1} d_{2}-c_{2} d_{1}=0
\end{aligned}
$$

denn der der reellen Achse der $z$ Ebene entspreehende Kreis hat die Gleichung:

$$
\begin{gathered}
\left(u^{2}+v^{3}\right)\left(c_{2} d_{1}-c_{1} d_{2}\right)-\left(a_{1} b_{2}-a_{2} b_{1}\right)+ \\
+u\left(c_{1} b_{2}+a_{1} d_{2}-c_{2} b_{1}-a_{2} d_{1}\right)+v\left(a_{1} d_{1}+a_{2} d_{2}-c_{1} b_{1}-c_{2} b_{2}\right)=0 .
\end{gathered}
$$

Man sieht, dass $a$ oder $b, c$ oder $d$ nicht verschwinden diurfen. Unterwirft man nun $z$ einer reellen Substitution:

$$
\left(z, f_{v}(z) \equiv\left(z, \frac{A_{\nu} z+B_{v}}{\overline{C_{\nu}} z+D_{v}}\right)\right.
$$

so ändert die entstehende Substitution:

oder:

$$
\left(\frac{a z+b}{c z+d}, \frac{a f_{v}(z)+b}{c f_{\nu}(z)+\bar{d}}\right)
$$

$\left(z, \frac{\left(-d\left(a A_{\nu}+b C_{\nu}\right)+c\left(a B_{\nu}+b D_{\nu}\right)\right) z+\left(b\left(a A_{\nu}+b C_{\nu}\right)-a\left(a B_{\nu}+b D_{\nu}\right)\right.}{\left(-d\left(c A_{\nu}+d C_{\nu}\right)+c\left(c B_{\nu}+d D_{\nu}\right)\right) z+\left(b\left(c A_{\nu}+d C_{\nu}\right)-a\left(c B_{\nu}+d D_{\nu}\right)\right)}\right)$ den Einheitskreis nicht.

Wir setzen hier und im folgenden

$$
a=i, b=1, c=1, d=i \text {, }
$$

wobei einem Punkte $z$ oberhalb der reellen Achse ein Punkt

$$
t=\frac{i z+1}{z+i}
$$

im Innern des Einheitskreises entspricht, und nunmehr lautet die letzte Substitution : 


$$
\left(z, \frac{\left(A_{\nu}+D_{\nu}+i\left(B_{\nu}-C_{\nu}\right)\right) z+\left(B_{\nu}+C_{\nu}+i\left(A_{\nu}-D_{\nu}\right)\right)}{\left(B_{\nu}+C_{\nu}-i\left(A_{\nu}-D_{\nu}\right)\right) z+\left(A_{\nu}+D_{\nu}-i\left(B_{\nu}-\overline{\left.C_{\nu}\right)}\right)\right.}\right),
$$

die wir noch so umformen, dass die Determinante Eins wird; d. h. wir dividieren alle Coefficienten durch $2 \sqrt{A_{\nu} D_{v}-B_{\nu} C_{\nu}}$.

\section{$\$ .2$.}

Angenommen, man habe aufgrund der Angaben des vorigen Paragraphen eine Gruppe aus $n$ Fundamentalsubstitutionen

$$
\left(z, f_{i}(z)\right)=\left(z, \frac{\alpha_{i} z+\beta_{i}}{\gamma_{i} z+\delta_{i}}\right) \quad\left(\alpha_{i} \delta_{i}-\beta_{i} \gamma_{i}=1\right),
$$

welche den Einheitskreis nicht alterieren, abgeleitet, so ist nunmehr die Aufgabe zu lösen: eindeutige, analytische Functionen $F(z)$ zu bilden, welche durch die verschiedenen Substitutionen der Grzppe nicht geändert werden.

Eine solche Function erhält offenbar jeden Wert, den sie überhaupt annimmt, an den Stellen eines Polygons, zu welchen die Stellen innerhalb der Begrenzung zu rechnen sind, ferner, die Stellen je einer Seite jedes Paares conjugierter Seiten und endlich je eine Ecke jedes Cyclus, doch zählt diese $p$ fach, wemn die Summe der Winkel an den Ecken des $m$ gliederigen Cyclus $\frac{2 \pi}{p}$ ist, oder - was dasselbe bedentet - wenn in jeder Ecke des Cyclus $p m$ Bereiche zusammenstoßen. Hat die Function $F(z)$ an einer solchen Ecke $z=\alpha$ den Wert $a$, muss sie in der Umgebung von $z=\alpha$ eine Entwicklung der Gestalt

$$
F(z)=a+a_{1}(z-\alpha)^{p}+a_{1}(z-\alpha)^{p+1}+\cdots
$$

besitzen, denn $F^{\prime}(z)$ durchlauft auf $p$ verschiedenen, aber congruenten, in $z=\alpha$ endigenden Wegen dieselbe Wertereihe.

Die Function nimmt aber auch jeden Wert an und wird an bestimmten Stellen eines Polygons von endlicher Ordnung Null und an anderen Stellen desselben Bereiches wie eine rationale Function unendlich. Die äquivalenten Stellen in dem Einheitskreise sind Null- und außerwesentlich singuläre Unendlichkeitsstellen gleicher Ordnung, aber die Punkte des Einheitskreises selbst werden wesentlich singuläre Stellen für $F(z)$.

Zur Herstellung einer Function $F(z)$ mit vorgegebenen Null- und Unendlichkeitsstellen bedarf man anderer Hilfsfunctionen als der $\Theta(z)$ Functionen, und zwar eindeutiger Functionen, bei welchen die als wesentlich verschieden gezählten Null- und Unendlichkeitsstellen eines Polygons stets ersichtlich bleiben. Wir wollen darum zunächst eine eindeutige Function bilden, welche innerhalb des Fundamentalpolygons $R_{0}$ die einfache Nullstelle $a$ and in dem aus $R_{0}$ durch die Substitution $\left(z, f_{i}(z)\right)$ unserer Gruppe hervorgehenden Polygone $R_{v}$ die einfache Null- 
stelle $f_{\nu}(a)$ besitzt; doch sei fürs erste weder $a$ noch $f_{\nu}(a)$ gleich Null. Ferner aber soll die Function innerhalb des Einheitskreises regulären Verhaltens und über denselben nicht fortzusetzen sein.

Wir haben in den Stellen

$$
a, f_{1}(a), f_{2}(a), \ldots f_{v}(a), \ldots
$$

eine unendliche Punktmenge $Q$, die in Verbindung mit der ersten abgeleiteten Punktmenge $Q^{\prime}$ einen zusammenbängenden Bereich begrenzt. Ordnen wir den genannten Stellen, die auch

$$
a_{0}, a_{1} ; a_{2}, a_{3}, \ldots, a_{y}, \ldots
$$

heißen mögen, neue Punkte

$$
b_{0}, b_{1}, b_{2}, b_{3}, \ldots b_{\gamma}, \ldots
$$

außerhalb des Einheitskreises oder auf demselben, zu und zwar so, dass nach Bezeichnung der unteren Grenze von $\left|b_{y}-b\right|$ mit $\beta_{\nu}$, wo unter $b$ irgend ein Punkt der Menge $Q^{\prime}$ verstanden ist,

$$
\lim _{\nu=\infty}\left(\left|a_{\nu}-b_{\nu}\right|-\beta_{\nu}\right)=0
$$

wird, oder so dass jeder positiven Größe $\eta$ eine solche positive ganze Zahl $n$ zugeordnet werden kann, dass für alle $\nu \geqq n$

$$
\left|a_{\nu}-b_{\nu}\right|-\beta_{\nu}<\eta
$$

ist und endlich die obere Grenze der $\beta_{\nu}$ eine endliche Größe $\beta$ ist, dann hat man in dem Product von Primfunctionen:

$$
\prod_{\nu=0}^{\infty}\left(1-\frac{a_{\nu}-b_{\nu}}{z-b_{\nu}}\right) c_{\mu=1}^{m_{\nu}} \frac{1}{\mu}\left(\frac{a_{\nu}-b_{\nu}}{z-h_{\nu}}\right)^{\mu}=\prod_{\nu=1}^{\infty} E_{\nu}\left(\frac{a_{\nu}-b_{\nu}}{z-b_{\nu}}\right)
$$

eine Function der verlangten Art, wenn nach Wahl einer unendlichen Folge positiver Größen $\varepsilon_{0}, \varepsilon_{1}, \varepsilon_{2}, \ldots \varepsilon_{\gamma}, \ldots$ von endlicher Summe und nach Wahl einer zweiten Folge positiven Größen $\xi^{(0)}, \varepsilon^{(1)}, \varepsilon^{(2)}, \ldots \ldots \varepsilon^{(\nu)}, \ldots$ mit der Grenzstelle $1\left(\lim \varepsilon^{(\nu)} \mp 1\right)$ $\nu=\infty$

über $m_{r}$ so verfügt worden ist, dass für die an die Bedingung $\left|\frac{a_{\nu}-b_{\nu}}{z-b_{\nu}}\right| \leqq \varepsilon^{(\nu)}$ geknüpften $z$ Werte

$$
\left|\sum_{\mu=m_{\nu}+1}^{\infty} \frac{1}{\mu}\left(\begin{array}{l}
a_{\nu}-b_{\nu} \\
z-b_{\nu}
\end{array}\right)^{\mu}\right|<\varepsilon_{\nu} .
$$

Ist $a_{v}=0$ und $b_{v}=\infty$, so setze man $E_{v}$ gleich z.

So lautet ein allgemeiner Satz von Herrn MittagL effle $\mathrm{r}$ ungeachtet eines besonderen Zusammenhanges zwischen den Stellen $\left.a_{i} .{ }^{*}\right)$ In unserem Falle ist die untere Grenze von

*) Acta mathematica. Bd. 4 . 
$\left|b_{\nu}-b\right|$ offenbar $\left|b_{\nu}\right|-1=\beta_{\nu}$. Die weitere Forderung an $q_{\nu}=b_{\nu}^{(1)}+i b_{\nu}^{(2)}$ gegenüber $a_{\nu}=a_{\nu}^{(1)}+i a_{\nu}^{(2)}$ lautet daher:

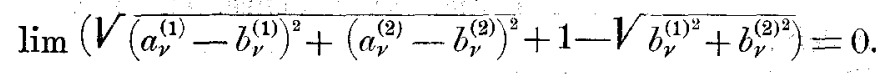

Doch weil $\lim \left(a_{\nu}^{(1)^{2}}+a_{\nu}^{(2)^{2}}\right)=\lim \left|a_{\nu}\right|^{2}=1$ ist, geht für $\lim b_{\nu}$ die Bedingung hervor:

$$
b_{\nu}^{(1)^{2}}\left(a_{\nu}^{(1)^{2}}-1\right)+b_{\nu}^{(2,2}\left(a_{\nu}^{(2)^{2}}-1\right)+2 a_{\nu}^{(1)} a_{\nu}^{(2)} b_{\nu}^{(1)} b_{\nu}^{(2)}=0 .
$$

Diese Gleichung für den Ort des Punktes $\left(b_{\nu}^{(1)}, b_{\nu}^{(2)}\right)$ stellt aber die durch die Punkte $z=0$ und $z=a_{\nu}$ gehende Doppelgerade dar.

Nach dieser Bemerkung erscheint es am naheliegendsten, jedem Punkt $a_{\nu}$ seinen symmetrischen

$$
\frac{1}{\bar{a}^{\prime} v^{\prime}}=\frac{a}{|a|^{2}}
$$

als Punkt $b_{x}$ zuzuordnen, was unter anderem den Vortheil gewährt, dass jetzt der durch eine Substitution $\left(z, f_{\mu}(z)\right)$ aus $b_{\nu}$ abgeleitete Punkt $f_{\mu}\left(b_{\nu}\right)$ mit dem zu $f_{\mu}\left(a_{\nu}\right)$ zuzuordnenden zusammenfällt. Es wird

$$
\left|a_{\nu}-b_{\nu}\right|=\frac{1-\left|a_{\nu}\right|^{2}}{\left|a_{\nu}\right|}, \beta_{\nu}=\frac{1-a_{\nu} \mid}{\left|a_{\nu}\right|},\left|a_{\nu}-b_{\nu}\right|-\beta_{\nu}=1-\left|a_{\nu}\right| .
$$

Wir treffen diese Wahl, werden aber das Zeichen $b_{y}$ beibehalten, und untersuchen nunmehr das obige Product mit Rücksicht darauf, dass die Stellen $a_{0}, a_{1}, \ldots a_{\nu}, \ldots$ die Transformierten einer unter ihnen sind.

Nehmen wir innerhalb des Einheitskreises eine von den Stellen $\alpha_{\gamma}$ verschiedene Stelle $z_{0}$ heraus, geben $z$ alle an die Bedingung $\left|z-z_{0}\right| \leqq \varrho$ geknüpften Werte, unter denen kein $a_{\nu}$ vorkommen soll, und lassen dann $\nu$ in dem Ausdruck $\left|z-b_{\nu}\right|-b_{\nu}$ die natürliche Zahlenreihe durchlaufen, so gibt es eine positive, untere Grenze $l$ für $\left|z--b_{\nu}\right|-\beta_{\nu}$. Es wird

$$
\mid z-b_{\nu} \geq \beta_{\nu}+l>0 \text {. }
$$

Da man aber jeder positiven Größe $\eta<l$ eine ganze, positive Zahl $n_{1}$, so zuordnen kann, dass für $\nu \geqq n_{1}$

ist, gelten die Ungleichungen

$$
\left|a_{\nu}-b_{\nu}\right|<\beta_{\nu}+\eta
$$

$$
\left|\frac{a_{\nu}-b_{\nu}}{z-b_{\nu}}\right|<\frac{\eta+\beta_{\nu}}{l+\beta_{\nu}}<\frac{\eta+\beta}{t+\beta}
$$

Weil noch $\beta$ endlich ist, kann man auch eine ganze Zahl $n_{2}$ so bestimmen, dass fïr $y \geq n_{2}$

$$
\frac{\eta+\beta}{l+\beta}<\varepsilon^{(v)}
$$

Nennt man $n$ die größere der Zahlen $n_{1}$ und $n_{2}$, ist für alle $\nu \geqq n$

$$
\left|\frac{a_{\nu}-b_{\nu}}{z-b_{\nu}}\right|<\varepsilon^{(\nu)}
$$


Wenn aber $\left|\frac{a_{\nu}-b_{\nu}}{z-b_{\nu}}\right|<1$ ist, gilt die Entwicklung

$$
E_{r}\left(\frac{a_{\nu}-b_{\nu}}{z-b_{\nu}}\right)=e^{-\sum_{\mu=m_{\nu}+1}^{\infty} \frac{1}{\mu}\left(\frac{a_{\nu}-b_{\nu}}{z-b_{\nu}}\right)^{\mu}}
$$

Wählt man deshalb $m_{\gamma}$, wie es der Mittag-Leffler'sche Satz vorschreibt, wird offenbar für jede positive ganze Zahl $\nu^{\prime}$

$$
\sum_{x=\nu}^{\nu+v^{\prime}}\left|\sum_{\mu=n_{\nu}+1}^{\infty} \frac{1}{\mu}\left(\frac{a_{x}-b_{x}}{z-b_{x}}\right)^{\mu}\right|<\delta
$$

gemacht werden können, wo $\delta$ willkürlich klein ist, und man darf

$$
\prod_{\nu=0}^{\infty} E_{\nu}\left(\frac{a_{\nu}-b_{\nu}}{z-b_{\nu}}\right)=e^{-\sum_{\nu=1}^{\infty} \sum_{\mu=m_{\nu}+1}^{\infty} \frac{1}{\mu}\left(\frac{a_{\nu}-l_{\nu}}{z-b_{\nu}}\right)^{\mu}}
$$

setzen. Das Product der Primfunctionen ist in der Umgebung $\varrho$ der Stellen $z_{0}$ gleichmäßig convergent.

Nach dieser wegen der Deutlichkeit absichtlich geführten Wiederholung des Beweises von Herrn Mittag-Leffler für seinen Satz, bemerken wir auch, dass für hinlänglich große $n$

$$
\begin{aligned}
& \sum_{\nu=n+1}^{\infty}\left|\sum_{\mu=m \nu+1}^{\infty} \frac{1}{\mu}\left(\frac{a_{\nu}-b_{\nu}}{z-b_{\nu}}\right)^{\mu}\right|<\sum_{\nu=n+1}^{\infty}\left|\frac{a_{\nu}-b_{\nu}}{z-b_{\nu}}\right|^{m_{\nu}+1} \frac{1}{1-\left|\frac{a_{\nu}-b_{\nu}}{z=b_{\nu}}\right|}< \\
&<\frac{1}{1-\frac{\eta}{l+\beta}} \sum_{\nu=n+1}^{\infty}\left|\frac{a_{\nu}-b_{\nu}}{z-b_{\nu}}\right|^{m_{\nu}+1}
\end{aligned}
$$

ist. Daher hängt die gleichmäßige Convergenz unseres Productes von der der Reihe

$$
\sum_{\nu=n+1}^{\infty}\left|\frac{a_{\nu}-b_{\nu}}{z-b_{\nu}}\right|^{m_{\nu}+1}
$$

ab. Weil aber für genügend große $n\left|z-b_{\nu}\right|$ in der Umgebung $\varrho$ von $z_{0} \geqq \beta+l$ ist; wird diese Reihe convergieren, sobald

convergent ist.

$$
\sum_{\nu=0}^{\infty}\left|a_{\nu}-b_{\nu}\right|_{\nu}^{m_{\nu}+1}
$$

Jetzt ist zu entscheiden, ob man in der letzten Reihe die Convergenz dadurch erzielen kann, dass man allen Zahlen $m_{r}$ 
ein und denselben Wert $m$ beilegt, ob also die Reihe

$$
\sum_{\nu=0}^{\infty}\left|a_{\nu}-b_{\nu}\right|^{m+1}
$$

für einen positiven, endlichen, ganzzahligen Wert von $m$ convergiert.

M. Poin caré nennt die Größe $L$ eines Bogens das längs dem Bogen erstreckte Integral $\int_{1} \frac{d z}{|z|^{2}}$, und die Größe $S$ einer Fläche, das über dieselbe ausgedehnte Doppelintegral $\iint \frac{\varrho d \varrho d v}{1-\varrho^{2}}$, wo $z=\rho e^{i w}$ gesetzt ist. Die Größe $L$ des Radius $\varrho$ eines um den Punkt $z=0$ gelegten Kreises ist $\frac{1}{2} \log \frac{1+\varrho}{1-\varrho}$, und man hat $\varrho=\frac{e^{2 L}-1}{e^{2 L}+1}$. Die Größe $S$ des Kreises ist

$$
\frac{\pi \varrho^{3}}{1-\varrho^{2}}=\frac{\pi}{4}\left(e^{2 L}+e^{-2 L}-2\right)
$$

und diese Größe ist $<\frac{\pi}{4} e^{2 L}$.

Denkt man um einen innerhalb des Einheitskreises befindlichen Punkt $z$ eine Kreisfläche $C_{o}$ gelegt, welche keinen äquivalenten Punkt im Innern oder auf ihrer Begrenzung enthält, bestimmen wir ihre Größe $S$ gleich $\sigma$, welche den Größen der congruenten Flächen um die $z$ entsprechenden Punkte $f_{v}(z)$ gleichkommt, legen alle Orthogonalkreise des Einheitskreises, bestimmen die Größe $L$ jedes durch $C_{o}$ ausgeschnittenen Bogens und nennen deren oberen Grenze $\lambda$, so ist die Zahl der $z$ äquivalenten Stellen innerhalb zweier Kreise $K_{n-1}$ and $K_{n}$ um den Punkt $z=0$, deren Radien $\varrho_{n-1}$ und $\varrho_{n}$, derart gewählt sind, dass die zugehörigen Größen $L$ :

$$
L_{n-1}=\frac{1}{2} \log \frac{1+\varrho_{n-1}}{1-\varrho_{n-1}}=(n-1) r, L_{n}=\frac{1}{2} \log \frac{1+e_{n}}{1-\varrho n}=n r
$$

werden, d. h. in arithmetischer Reihe wachsen, kleiner als

$$
\frac{\pi}{4 \sigma}\left(e^{2(n r+\lambda)}+e^{-2(n r+\lambda)}-2\right)<\frac{\pi}{4 \sigma} e^{2(n r+\lambda)} .
$$

Verwenden wir diesen Satz, ersetzen aber in

$$
\sum_{v}\left|a_{v}-b_{v^{\prime}}\right|^{n+1}=\sum_{v}\left(\frac{1-\mid a_{v}{ }^{2}}{\left|a_{v}\right|}\right)^{m+1}
$$

die Beträge $\left|a_{v}\right|$, welche $>\varrho_{n-1}$ aber $\leq \varrho_{n}$ sind, durch den kleineren Betrag $\varrho_{n-1}$, wobei das einzelne Glied größer wird, was immer durchgeführt werden soll außer bei den innerhalb des ersten Kreises liegenden Stellen, die wir nämlich bei dieser Untersuchung gang außeracht lassen können, weil sie nur in 
endlicher Anzahl vorkommen, - dann folgt die Ungleichung:

$$
\mathbf{\Sigma}^{\prime}\left|a_{\nu}-b_{\nu}\right|^{m+1}<\frac{\pi}{4 \sigma} e^{2 \lambda} \sum_{n=2}^{\infty} e^{2 n r}\left[\frac{1-\left|\frac{e^{2(n-1) r}-1}{e^{2(n-1) r}-1}\right|^{2}}{\left|\frac{e^{2(n-1) r}-1}{e^{2(n-1) r}-1}\right|}\right]^{m+1}
$$

oder

$$
\mathbf{\Sigma}^{\prime}\left|a_{r}-b_{r}\right|^{m+1}<\frac{4^{1 n} \pi}{\sigma} e^{2 \lambda} \sum_{n=2}^{\infty} e^{2 r} \frac{e^{2(n-1) r}}{\left(e^{2(n-1) r}-e^{-2(n-1) r}\right)^{m+1}} .
$$

Bezeichnet man $e^{2 r}$ mit $u$, so handelt es sich nur mehr um die Frage, für welchen Wert von $m$ die Reihe

$$
\sum_{\mu=1}^{\infty} \frac{u}{u^{\mu / n}} \frac{1}{\left(1-\frac{1}{u^{2 \mu}}\right)^{m+1}}
$$

convergieren kann. Weil diese Reihe eine kleinere Summe hat als

$$
\frac{u}{u^{m}}\left(1-\frac{1}{u^{2}}\right)^{-(m+1)}\left(1-\frac{1}{u^{m}}\right)^{-1} \text {, }
$$

indem zufolge der Bedeutung von $r u>1$ ist, besteht die Convergenz schon für den Fall $m=1$.

Darnach ist

$$
\prod_{\nu=0}^{\infty}\left(1-\frac{a_{\nu}-b_{\nu}}{z-b_{\nu}}\right) e^{\frac{a_{\nu}-b_{\nu}}{z-b_{\nu}}}=\tau\left(z ; a_{0}\right)
$$

eine der zu Beginn des Paragraphen verlangten Functionen, und jede andere geht durch Multiplication mit einer willkürlichen Function der Form $e^{G(z)}$ hervor, wenn $G(z)$ eine innerhalb des Einheitskreises durchaus reguläre und eindentige Function bedeutet.

Es ist klar, dass bei Vertauschung von $a_{\nu}$ mit $b_{v}$ die außerhalb des Einheitskreises reguläre, eindeutige Function

$$
\prod_{\nu=0}^{\infty}\left(1-\frac{b_{\nu}-a_{\nu}}{z-a_{\nu}}\right) e^{\frac{b_{\nu}-a_{\nu}}{z-a_{\nu}}}=\tau\left(z ; b_{0}\right)
$$

mit den einfachen Nullstellen $b_{v}, b_{1}, \ldots b_{\nu}, \ldots$ entspringt.

Heben wir die frühere Beschränkung über die Lage der Stellen $a_{0}, a_{1}, \ldots a_{v}, \ldots$ innerhalb der Polygone auf und nehmen an, dass die Stellen $f_{\nu}(a)$ mit den $p$-fach zu zählenden Ecken eines $m$-gliederigen Cyclus zusammenfallen, so ist die $\tau\left(z ; a_{n}\right)$ analoge Function:

$$
\prod_{r=0}^{\infty}\left(1-\frac{a_{\nu}-b_{\nu}}{z-b_{\nu}}\right)^{p \frac{a_{\nu}-b_{\nu}}{z-b_{\nu}}}
$$


Ist ferner eine der Stellen $a_{r}$ innerhalb eines Polygons Null, worauf die durch die Substitution $\left(z, \frac{\alpha_{\nu} z+\beta_{\nu}}{\gamma_{\nu} z+\delta_{\nu}}\right)$ transformierte Stelle $a_{v}=\frac{\beta_{\nu}}{\delta_{v}}$ ist, so haben wir $a=o, b=\infty$ und $a_{v}=\frac{\beta_{\nu}}{\delta_{\nu}}$ die durch die obige Substitution der Stelle $\infty$ entsprechende Stelle $b_{\nu}=\frac{a_{\nu}}{\gamma_{\nu}}$ zuzuordnen. Es soll aber $b_{\nu}=\frac{1}{a_{\nu}^{\gamma}}=\frac{a_{\nu}}{\left|a_{\nu}\right|^{2}}$ sein. Diese Forderung ist gemäß der allgemeinen Form unserer Substitutionen (auf Seite 10) erfüllt, es ist

$$
{ }_{\nu_{\nu}}^{\alpha_{\nu}}=\frac{A_{\nu}+D_{\nu}+i\left(B_{\nu}-C_{\nu}\right)}{B_{\nu}+C_{\nu}-i\left(A_{\nu}-\overline{D_{\nu}}\right)}=\frac{1}{\left(\frac{\beta_{\nu}}{\delta_{\nu}}\right)^{\prime}}=\frac{1}{\left(\frac{B_{\nu}+C_{\nu}+i\left(A_{\nu}-D_{\nu}^{\nu}\right)}{A_{\nu}+D_{\nu}-i\left(\bar{B}_{\nu}-C_{\nu}\right)}\right)} .
$$

Es wird darnach:

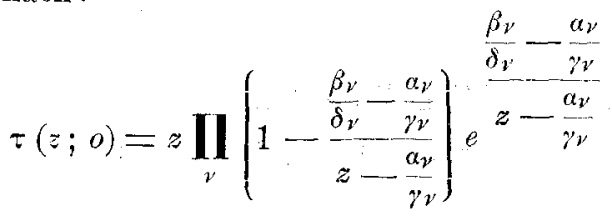

und

$$
\tau(z ; \infty)=\frac{1}{z} \prod_{\nu}\left(1-\frac{\frac{\alpha_{\nu}}{\gamma_{\nu}}-\frac{\beta_{\nu}}{\delta_{\nu}}}{z-\frac{\beta_{\nu}}{\delta_{\nu}}}\right) e^{\frac{\alpha_{\nu}-\frac{\beta_{\nu}}{\gamma_{\nu}}}{z-\frac{\beta_{\nu}}{\delta_{\nu}}}}
$$

Die oben erwiesene Convergenz der Reihe $\underset{\nu}{\mathbf{\Sigma}}\left|a_{\nu}-b_{\nu}\right|^{2}$ elscheint auffallend, wenn man die Convergenz, beziehungsweise Divergenz der Reihen $\Sigma^{\prime}\left|a_{\nu}\right|^{-3}, \Sigma^{\prime}\left|a_{\nu}\right|^{-2}$ beachtet, wo $a_{\nu}-$ bis auf $z=0$ - die Nullstellen $2 \mu \omega+2 \mu^{\prime} \omega^{\prime}\left(\mu, \mu^{\prime}=0, \pm 1, \pm 2, \ldots\right)$ der in der Weierstra B'schen Theorie der doppeltperiodischen Functionen benuitzten Hilfsfunction $\sigma(z)$ bedenten. Hier ist jeder Stelle $a_{\nu}$ die Stelle $b_{\nu}=\infty$ zugeordnet, und die Substitutionen $\left(z, f_{\nu}(z)\right)$ sind $\left(z, z+a_{\nu}\right)$.

Wir sehen nach, wo unser Convergenzbeweis in dem Ausnahmsfalle eine Änderung erheischt. Die für Bogen und Flächen gegenüber den Substitutionen bestehenden Invarianten $L$ und $S$ sind hier

$$
L=\int|d z|, S=\iint d x d y
$$

daher ist die Größe $L$ des Radius $\varrho$ eines um den Punkt $z=0$ beschriebenen Kreises $\varrho$ selbst, und die Größe $S$ der Kreisfläche ist $\varrho^{2} \pi$. Beschreibt man um eine Stelle $z$ einen Kreis, der keine $z$-äquivalente Stelle im Innern oder auf seiner Begrenzung besitzt, heiß̈t seine Fläche $\sigma$, ist ferner $\lambda$ der Durchmesser dieser Fläche, 
dann wird die Zabl der in dem Kreise $\varrho$ um $z=0$ befindlichen transformierten Stellen von $z$ kleiner sein als $\frac{\pi(\varrho+\lambda)^{2}}{\sigma}$. Legt man jetzt Kreise $K_{1}, K_{2}, \ldots K_{n}, \ldots$ mit den Radien $r, 2 r, \ldots n r, \ldots$ um den Punkt $z=0$, ersetzt jeden Wert $\left|a_{y}\right|$ außerhalb des $(n-1)^{\text {ten }}$ und nicht außerhalb des $n^{\text {ten }}$ Kreises durch den kleineren Betrag $(n-1) r$, auf dass

$$
\left|\frac{1}{a_{\nu}}\right|^{\mid m+1}<\left|\frac{1}{(n-1) r}\right|^{m+1}
$$

ist, und bezeichnet endlich $\lambda$ mit $k r$, dann gilt Umgleichung

$$
\sum_{v}\left|\frac{1}{a_{\nu}}\right|^{m+1}<\frac{\pi}{\sigma} \sum_{n} \frac{(n r+k r)^{2}}{((n-1) r)^{m+1}}=\frac{\pi}{\sigma r^{m-1}} \sum_{n} \frac{(n+k)^{2}}{(n-1)^{m+1}},
$$

wo die Summation von $n=2$ bis $n=\infty$ zu erstrecken ist, wenn man wieder die in und auf dem ersten Kreise $K_{1}$ befindlichen Stellen $a_{v}$, welche nur in endlicher Anzahl vorkommen, ausschließt.

Die für die Convergenz der erhaltenen Reihe nothwendige Bedingung, dass das allgemeine Glied für $n=\infty$ den Grenzwert Null hat, ist offenbar erst erfüllt, wenn $m=2$ ist, und das steht mit der Divergenz von $\left.\underset{\nu}{\Sigma^{\prime}} \mid a_{\nu}\right\}^{-2}$ in Einklang.

Bevor wir unsere Function $\tau\left(z ; a_{0}\right)$ weiterhin untersuchen, bilden wir gelegentlich andere eindeutige Functionen von Bedeutung; zunächst eine Function, welche innerhalb des Einheitskreises die Stellen $a_{v}$ zu außerwesentlich singulären hat und in deren Umgebung die Entwicklungsform besitzt:

$$
\frac{b_{\nu}-a_{\nu}}{z-a_{\nu^{\prime}}}+\mathfrak{B}_{\nu}\left(z-a_{\nu}\right) \text {. }
$$

Für Werte von $z$, welche an die Bedingung $\left|\frac{a_{\nu}-b_{v}}{z-b_{\nu}}\right|<1$ geknüpft sind, gilt die Entwicklung:

Bildet man

$$
\frac{b_{\nu}-a_{\nu}}{z-a_{\nu}}=-\sum_{\mu=1}^{\infty}\left(\frac{a_{\nu}-b_{v}}{z-b_{\nu}}\right)^{\mu} .
$$

$$
F_{\nu}(z)=\frac{b_{\nu}-a_{\nu}}{z-a_{\nu}}+\sum_{\mu=1}^{m_{\nu}}\left(\frac{a_{\nu}-b_{\nu}}{z-b_{\nu}}\right)^{\mu}
$$

für den Fall $m_{y}=1$, auf dass

entsteht, ist

$$
F_{\nu}(z)=\left(b_{\nu}-a_{\nu}\right)\left(\frac{1}{z-a_{v}}-\frac{1}{z-b_{\nu}}\right)
$$

$$
F(z)=\sum_{\nu} F_{\nu}(z)=\sum_{\nu}\left(b_{\nu}-a_{\nu}\right)\left(\frac{1}{z-a_{\nu}}-\frac{1}{z-b_{\nu}}\right)
$$

die verlangte Eunction, denn die Reihe 
ist convergent.

$$
\sum_{\mid}\left|F_{\nu}(z)\right| \leqq \sum_{\nu} \sum_{\mu=1}^{\infty}\left|\frac{a_{\nu}-b_{\nu}}{z-b_{\nu}}\right|^{\mu+1}
$$

Construiert man diejenige eindeutige Function, welche außerhalb des Einheitskreises die Stellen $b_{\nu}$ zu außerwesentlich singulären hat, und in der Umgebung von $b_{r}$ eine Darstellung der Form

$$
\frac{a_{\nu}-b_{\nu}}{z-b_{\nu}}+\mathfrak{P}^{(v)}\left(z-b_{v}\right)
$$

zulässt, gelangt man zu dem arithmetisch gleichartig gebildeten Ausdruck

$$
\sum_{\nu}\left(a_{\nu}-b_{\nu}\right)\left(\frac{1}{z-b_{\nu}}-\frac{1}{z-a_{\nu}}\right) .
$$

Darum stellt derselbe Ausdruck außerhalb und innerhalb des Einheitskreises je eine analytische Function dar.

Eine andere wichtige Function geht durch logarithmische Differentiation von $\tau\left(z ; a_{0}\right)$ hervor, und zwar:

$$
\frac{\tau^{\prime}\left(z ; a_{o}\right)}{\tau\left(z ; a_{o}\right)}=\sum_{\nu} \frac{a_{\nu}-b_{\nu}}{z-b_{\nu}}\left(\frac{1}{z-a_{\nu}}-\frac{1}{z-b_{\nu}}\right) .
$$

Sie lässt sich auch finden, indem man eine Function fordert, welche in der Umgebung von $a_{y}$, die Darstellungsform

$$
\frac{1}{z-a_{\nu}}+\mathfrak{B}_{\nu}\left(z-a_{\nu}\right)
$$

besitzt, oder in der Umgebung von $b_{\gamma}$ die Entwicklung zulässt:

$$
\frac{1}{z-b_{\nu}}-\frac{a_{\nu}-b_{\nu}}{\left(z-b_{\nu}\right)^{3}}+\mathfrak{p}^{(\nu)}\left(z-b_{\nu}\right) \text {. }
$$

Man hat bei der Bildung von $F_{\nu}(z)$, die entsprechend dem früheren Falle vorzunehmen ist, nur $m_{y}=2$ zu setzen.

Jetzt gehen wir zu der Function $\tau\left(z ; \alpha_{o}\right)$ zurück und sehen nach, wie sie sich gegenüber einer Substitution der Gruppe

verhält.

$$
(z, f(z))=\left(z, \frac{\alpha z+\beta}{\gamma z+\delta}\right) \quad(\alpha \delta-\beta \gamma=1)
$$

Wir notieren die Beziehungen:

$$
\left(z, f^{-1}(z)\right)=\left(z, \frac{-\delta z+\beta}{\gamma z-a}\right), \quad f(z)-\mathrm{c}=\frac{a-c \gamma}{z \gamma+\delta}\left(z-f^{-1}(c)\right),
$$

und bemerken, dass die Gleichung besteht:

$$
1-\frac{a_{\nu}-b_{\nu}}{f(z)-b_{\nu}}=\frac{1-\frac{f-1\left(\sigma_{\nu}\right)-f-1\left(b_{\nu}\right)}{z-y-1\left(b_{\nu}\right)}}{1-\frac{b_{\nu}-a_{\nu}}{\frac{a}{\gamma}-a_{\nu}}}
$$


Setzt man

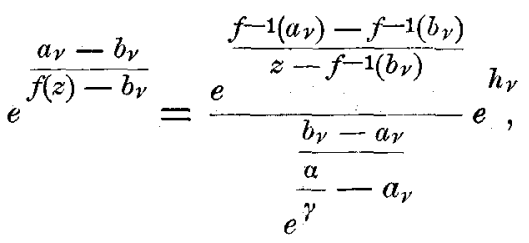

wird

oder

$$
h_{\nu}=\left(\mathrm{z}-\left(-\frac{\delta}{\gamma}\right)\right) \frac{a_{\nu}-b_{\nu}}{z-f^{-1}\left(b_{\nu}\right)}\left(\frac{1}{\frac{\alpha}{\gamma}-b_{\nu}}-\frac{1}{\frac{\alpha}{\gamma}-a_{\nu}}\right)
$$

$$
h_{\nu}=\frac{a_{\gamma}-b_{\nu}}{f(z)-\iota_{\nu}} \frac{b_{\nu}-\alpha_{\nu}}{\frac{a}{\gamma}-\alpha_{\nu}} .
$$

Wir werden fernerhin zur näheren Bezeichnung der Function $h_{\nu}$ ausführlicher schreiben $h_{\nu}\left(f(z), \frac{\alpha}{\gamma} ; a_{\nu}\right)$ oder $h_{\nu}\left(f(z), f(\infty) ; a_{\nu}\right)$

Weil nun die Punktmenge $f^{-1}\left(a_{\nu}\right) \quad(\nu=0,1,2,3 \ldots)$ von der gegebenen $\left(a_{0}, a_{1}, a_{2}, \ldots\right)$ nur in der Anordnung abweicht, ist zunächst auch

$$
\tau\left(z ; a_{0}\right)=\prod_{\nu}\left(1-\frac{f^{-1}\left(a_{\nu}\right)-f^{-1}\left(b_{\nu}\right)}{z-f^{-1}\left(b_{\nu}\right)}\right) e^{\frac{f^{-1}\left(a_{\gamma}\right)-f^{-1}\left(b_{\nu}\right)}{z-f^{-1}\left(b_{\nu}\right)}}
$$

und man kann hierauf

$\left.\tau\left(f(z) ; a_{0}\right)=\frac{\tau\left(z ; a_{0}\right)}{\tau\left(\frac{\alpha}{\gamma} ; b_{0}\right)} e^{\left(z-\left(-\frac{\delta}{\gamma}\right)\right) \frac{a_{\nu}-b_{\nu}}{z-f^{-1}\left(b_{\nu}\right)}\left(\frac{1}{\gamma}-b_{\nu}\right.}-\frac{1}{\frac{\alpha}{\gamma}-a_{\nu}}\right)$

setzen, sobald nur nachgewiesen ist, dass die in der Exponentialfunction stehende Reihe gleichmäßig convergiert und $\left|z-\left(-\frac{\delta}{\gamma}\right)\right|$ nicht unendlich wird.

Da oben die gleichmäßige Convergenz von $F(z)$ in der Umgebung der außerhalb des Einheitskreises liegenden Stellen, welche von den Stellen $b_{v}$ verschieden sind, bewiesen wurde, und $z=\frac{\alpha}{\gamma}$ die durch die Substitution $(z, f(z))$ transformierte Stelle von $z=\infty$ ist, also $\left|\frac{\alpha}{\gamma}\right|>1$ ist und $\frac{\alpha}{\gamma}$ niemals einem $b_{\nu}$ gleichkommt, convergiert

$$
\sum_{\nu}\left|a_{\nu}-b_{\nu}\right|\left|\frac{1}{\frac{\alpha}{\gamma}-b_{\nu}}-\frac{1}{\frac{a}{\gamma}-a_{\nu}}\right| .
$$

Ferner ist $\left|z-f^{-1}\left(b_{v}\right)\right|$ in der Umgebung $\varrho$ jeder innerhalb des Monatsh. f. Mathematik u. Physik. I, Jahrg., 2. Heft. 
Einheitskreises befindlichen Stelle endlich und von Null verschieden. Dadurch ist die Convergenz der obigen Reihe bewiesen.

Zufolge der im Paragraphen 1 getroffenen Verfügungen hat jede lineare Substitution, welche den Einheitskreis nicht ändert und durch den Wert $\gamma=0$ ausgezeichnet ist, die Gestalt

Die Primfunction

$$
\left(z, \frac{\frac{A+i B}{\sqrt{A^{2}+B^{2}}} z}{\frac{A-i B}{V^{A^{2}+B^{2}}}}\right) \equiv\left(z, \frac{e^{i \varphi} z}{e^{-i \varphi}}\right) \equiv(z, \psi(z)) \text {. }
$$

$$
\left(1-\frac{a_{\nu}-b_{\gamma}}{z-b_{v}}\right) e^{\frac{a_{\gamma}-b_{\nu}}{z-b_{\nu}}}
$$

geht bei Anwendung einer solchen Substitution in die folgende über

$$
\left(1-\frac{\psi^{-1}\left(a_{\nu}\right)-\psi^{-1}\left(b_{\gamma}\right)}{z-y^{-1}\left(b_{\gamma}\right)}\right) e^{\frac{\psi^{-1}\left(a_{\nu}\right)-\psi^{-1}\left(b_{\gamma}\right)}{z-\psi^{-1}\left(b_{\gamma}\right)}},
$$

ohne dass ein Factor $e^{h}$ hinzutritt. Demnach besteht die Gleichung

$$
\tau\left(\psi(z) ; a_{0}\right)=\tau\left(z ; a_{0}\right) \text {. }
$$

Bei Substitutionen $(z, f(z))$ hingegen, in welchen $\gamma$ von Null verschieden ist, drückt nach Bezeichnung von $\Sigma h_{\gamma}$ mit $h(f(z)$, $f(\infty) ; a_{0}$ ) die Gleichung

$$
\tau\left(f(z) ; a_{0}\right)=\frac{\tau\left(z ; a_{0}\right)}{\tau\left(\frac{a}{\gamma} ; b_{0}\right)} e^{\left.h f(z), f(\infty) ; a_{0}\right)}
$$

das Verhalten der Fuuction $\tau\left(z ; \boldsymbol{a}_{0}\right)$ gegenüber der Substitution aus. $\left.{ }^{*}\right)$

Wir haben auch einen Ausdruck für $\tau(f(z) ; o)$ abzuleiten. Hierzu beachte man, dass

$$
f(o)=\frac{\beta}{\delta}, f(\infty)=\frac{\alpha}{\gamma}, f^{-1}(o)=-\frac{\beta}{\alpha} ; f^{-1}(\infty)=-\frac{\delta}{\gamma}
$$

ist und die Beziehungen bestehen :

$$
\begin{aligned}
& f(z)=\frac{1-\frac{f^{-1}(o)-f^{-1}(\infty)}{z-f^{-1}(\infty)}}{\frac{1}{\frac{\alpha}{\gamma}}} ; 1-\frac{f(o)-f(\infty)}{f(z)-f(\infty)}=\frac{1-\frac{f^{(o)}(o)-f^{(o)}(\infty)}{z-f^{\prime(o)}(\infty)}}{-\frac{\delta}{\gamma}}=\frac{z}{\delta} . \\
& *)\left(f(z), f(\infty) ; a_{0}\right) \text { kann man auch in der Form } \sum_{\nu} \frac{a_{\nu}-b_{\nu}}{f(z)-b_{\nu}} \frac{b_{\nu}-a_{\nu}}{\frac{\alpha}{\gamma}-b_{\nu}} \text { in }
\end{aligned}
$$

Rechnung bringen, denn die Convergenz dieser Reihe besteht anch. 
Darnach wird

$$
\begin{aligned}
& \tau(f(z) ; o)=\frac{\tau(z ; o)}{-\frac{\delta}{\gamma}\left(\tau\left(\frac{\alpha}{\gamma} ; \infty\right)\right)^{\prime}} \times \\
& \quad \times e^{\left.\left(z-\left(-\frac{\delta}{\gamma}\right)\right) \mathbf{\Sigma}_{\nu}^{\prime} \frac{\beta_{\nu}}{z-\frac{\alpha_{\nu}}{\delta_{\nu}}}-\frac{\alpha^{-1}\left(\frac{\alpha_{\nu}}{\gamma_{\nu}}\right)}{\left(\frac{\alpha}{\gamma}-\frac{\alpha_{\nu}}{\gamma_{\nu}}\right.}-\frac{1}{\frac{\alpha}{\gamma}-\frac{\beta_{\nu}}{\delta_{\nu}}}\right)+g(z),}
\end{aligned}
$$

wo die Striche bei dem Producte $\left(\tau\left(\frac{a}{\gamma} ; \infty\right)\right)$ und dem Summenzeichen anzeigen, dass $\alpha_{\nu}, \beta_{\nu}, \gamma_{\nu}, \delta_{\gamma}$ niemals mit $\alpha, \beta, \gamma, \delta$ übereinstimmen sollen und unter dem Summenzeichen nicht $\alpha_{\nu}=1$, $\beta_{\nu}=0, \gamma_{\nu}=0, \delta_{\nu}=1 \mathrm{zu}$ setzen ist. Die Funetion $g(z)$ ist gleich:

$$
-\frac{f^{-1}(o)-f^{-1}(\infty)}{z-f^{-1}(\infty)}+\frac{f^{\prime}(o)-f(\infty)}{f(z)-f(\infty)}=-\frac{1}{\alpha \gamma} \frac{1}{z-\left(-\frac{\delta}{\gamma}\right)}+\frac{\gamma}{\delta}\left(z-\left(-\frac{\delta}{\gamma}\right)\right) .
$$

Denken wir nun in dem Polygon $R_{v}$ des regulär eingetheilten Einheitskreises die Stellen

$$
c_{\nu}^{(1)}, c_{\nu}^{(2)}, \ldots c_{\gamma^{\prime}}^{(1)} ; \mathfrak{c}_{\nu^{\prime}}^{(1)}, \mathfrak{c}_{\nu}^{(2)}, \ldots \mathfrak{c}_{\nu}^{(1)}
$$

gegeben, nennen die symmetrischen Stellen

$$
d_{\nu}^{(1)}, d_{\nu}^{(2)}, \ldots d_{\nu}^{(k)} ; \mathfrak{d}_{\nu}^{(1)}, \mathfrak{b}_{\nu^{\prime}}^{(2)}, \ldots \mathfrak{d}_{v}^{(1)}
$$

und bezeichnen mit $G(z)$ eine innerhalb des Einheitskreises bestehende analytische Function, dann ist

$$
F(z)=\frac{\prod_{k=1}^{k} \tau\left(z ; c_{0}^{(k)}\right)}{\prod_{\lambda=1}^{l} \tau\left(z ; c_{j}^{(\lambda)}\right)} e^{G(z)}
$$

der Ausdruck fïr die allgemeinste innerhalb des Einheitskreises giltige eindeutige analytische Function mit den vorgegebenen Null- und Unendlichkeitsstellen

beziehungsweise $\left.\begin{array}{r}c_{v}^{(\lambda)}(x=1,2, \ldots k) \\ c_{\nu}^{(\lambda)}(\lambda=1,2, \ldots l)\end{array}\right\}(v=0,1,2, \ldots)$

$$
\left.\begin{array}{l}
c_{v}^{(x)}(x=1,2, \ldots l) \\
\mathfrak{c}_{\nu}^{(\lambda)}(\lambda=1,2, \ldots l)
\end{array}\right\}(v=0,1,2, \ldots)
$$

$C$ bezeichnet eine Constante.

Unterwirft man in $F(z)$ das Argument einer Substitution (z. $f(z))$ der vorgegebenen Gruppe und dividiert $F(f(z))$ durch $F(z)$, folgt die Beziehung

$$
\frac{F(f(z))}{F(z)}=\frac{\prod_{z=1}^{l} \tau\left(\frac{\alpha}{\gamma} ; b_{o}^{(\lambda)}\right)}{\sum_{x=1}^{k} \tau\left(\frac{\alpha}{\gamma}\right) ; d_{0}^{(x)}} \frac{e^{x} h\left(f(z) ; f(\infty) ; c_{0}^{(\alpha)}\right)}{\sum^{\lambda} h\left(f(z) ; f(\infty) ; c_{0}^{(\lambda)}\right)} e^{G(f(z))-G(z)},
$$


in welcher man zufolge der Gleichung:

$$
\begin{aligned}
& \prod_{\nu=0}^{\infty} \mathrm{E}_{\nu}\left(\frac{b_{\nu}-a_{\nu}}{z-a_{\nu}}\right)=e^{-\sum_{\nu=0}^{\infty} \sum_{\mu=2}^{\infty} \frac{1}{\mu}\left(\frac{b_{\nu}-a_{\nu}}{z-a_{\nu}}\right)^{\mu}}
\end{aligned}
$$

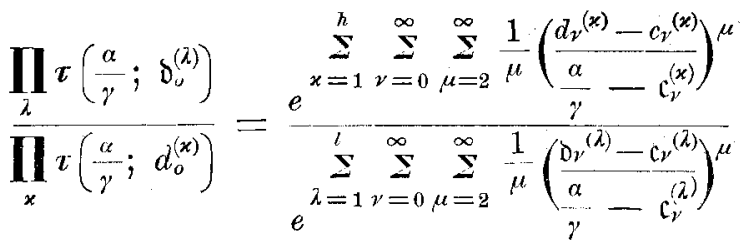

setzen darf. Im folgenden werden wir diesen Ausdruck mit

$$
e^{\sum_{x=1}^{l} M_{x}(f(\infty))-\sum_{\lambda=1}^{\infty} \mathfrak{M}_{\lambda}(f(\infty))}
$$

bezeichnen.

Für $G(z)$ kann man z. B. die Form gebrauchen:

wo

$$
G(z)=\sum_{x=1}^{k} G_{x}(z)-\sum_{\lambda=1}^{l}\left(B_{2}(z),\right.
$$

$$
G_{x,}(z)=\sum_{\nu=0}^{\infty} \sum_{\mu=1}^{\infty} \frac{A_{x \mu}^{(\nu)}}{\mu}\left(\frac{c_{\nu}^{(x)}-d_{\nu}^{(x)}}{z-d_{\nu}^{(x)}}\right)^{\mu}, \quad \lambda(z)=\sum_{\nu=0}^{\infty} \sum_{\mu=1}^{\infty} \frac{\Re^{(\nu)}}{\mu}\left(\frac{c_{\nu}^{(\lambda)}-\delta_{\nu}^{(\lambda)}}{z-\delta_{\nu}^{(\lambda)}}\right)^{\mu}
$$

ist. Bringt man

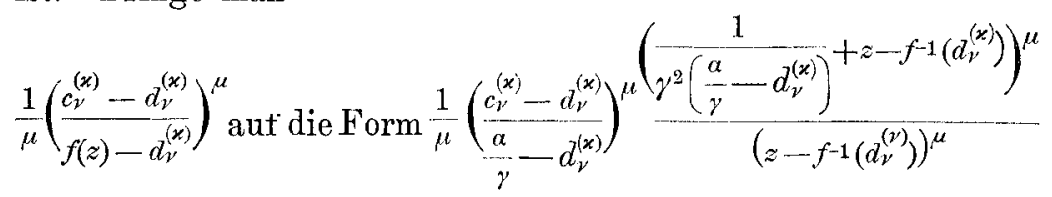
und versteht unter $A_{x \mu}^{(\nu-1)}$ den bei dem Gliede

$$
\frac{1}{\mu}\left(\frac{f-1\left(c_{\nu}^{(x)}\right)-f^{-1}\left(d_{\nu}^{(x)}\right)}{z-f^{-1}\left(d_{\nu}^{(x)}\right)}\right)^{\mu}
$$

stehenden Coefficienten $A$, wird der Coefficient von $\left(z-f^{-1}\left(d_{r}^{(x)}\right)\right)^{-m}$ der Entwicklung von $G_{x}(f(z))$ in der Umgebung der Stelle $f^{-1}\left(d_{y}^{(x)}\right)$

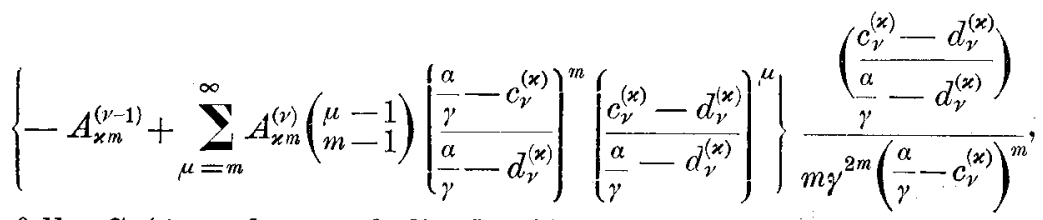

falls $G_{x}(z)$ auch außerhalb des Einheitskreises eine analytische Function ist. 
Verlangen wir, dass $F(z)$ durch die Fundamentalsubstitutionen $(z, f(z))(i=1,2, \ldots n)$ keine Änderung erfahre und damit auch ungeändert bleibe bei irgend einer anderen Substitution der zugehörigen Gruppe, müssen die $n$ Beziehungen bestehen:

$$
\begin{aligned}
& \sum_{k=1}^{l_{k}} M_{\chi}\left(f_{i}(\infty)\right)-\sum_{\lambda=1}^{l} \mathfrak{M}_{\lambda}\left(f_{i}(\infty)\right)+\sum_{k=1}^{k} h\left(f_{i}(z) ; f_{i}(\infty) ; c_{o}{ }^{(k)}\right)- \\
& -\sum_{\lambda=1}^{l} h\left(f_{i}(z) ; f_{i}(\infty) ; \mathfrak{c}_{0}{ }^{(\lambda)}\right)+G\left(f_{i}(z)\right)-G(z)=2 m_{i} \pi V^{\prime-1},
\end{aligned}
$$

wo $m_{i}$ ganze Zahlen bedeuten; die links stehenden Functionen haben in dem Einheitskreise die constanten Werte $2 m_{i} \tau V-1$ anzunehmen. Entwickelt man die genannten Functionen in der Umgebung der Stelle $z=0$ und erfüllt die hervorgehenden Gleichungen identisch, entstehen für die Coefficienten von $G(z)=\sum_{\mu=1}^{\infty} C_{\mu} z^{\mu} n$ Systeme von Gleichungen. Bei Untersuchung derselben hat man aber noch weitere Relationen in Rücksicht zu ziehen. Wenn man nämlich auf $\tau\left(z ; a_{0}\right) \operatorname{oder} \tau(z ; o)$ successive die Substitutionen anwendet, welche in einem der Fundamentalrelationen der Gruppe:

eintreten, wo

$$
z=f j_{1}\left(f j_{i}\left(\ldots f j_{s}(z)\right)\right)
$$

$$
\left(z ; f j_{1}(z), f j_{2}(z), \ldots f j_{s}(z)\right)
$$

nur Fundamentalsubstitutionen sind, muß ja

sein. Indem aber

$$
\tau\left(f j_{1}\left(f j_{2}\left(\ldots f j_{s}(z)\right)\right) ; a_{0}\right) \equiv \tau\left(z ; a_{0}\right.
$$

$$
=\frac{\tau\left(f j_{1}\left(f j_{2}\left(\ldots f j_{s}(z)\right)\right) ; a_{o}\right)=}{\prod_{\sigma=1}^{3} \tau\left(f j_{\sigma}(\infty) ; a_{o}\right)} e^{\sum_{\sigma=1}^{3} h\left(f j_{s}-\sigma+1\left(f j_{s-\sigma}\left(\ldots f j_{s}(z)\right)\right) ; f j_{s}-\sigma+1 ; a_{o}\right)}
$$

zu setzen ist, erhält man die von dem Werte $a_{0}$ nothwendig unabhängige Beziehung:

$$
\begin{gathered}
\left.\sum_{\sigma=1}^{3} h\left(f j_{s-\sigma+1}\left(f j_{s-\sigma}\left(\ldots f j_{\sigma} z\right)\right)\right) ; f_{j_{3-\sigma+1}}^{(\infty)} ; a_{o}\right)-\sum_{\sigma=1}^{3} \sum_{v=0}^{\infty} \sum_{\mu=2}^{\infty} \frac{1}{\mu}\left(\frac{a_{\nu}-b_{v}}{f j_{\sigma}^{(\infty)}-b_{v}}\right)^{\mu}= \\
=2 k \pi \sqrt{-1},
\end{gathered}
$$

in der $k$ wieder eine ganze Zahl ist. - Bei $q$ Cyclen gibt es q) soleche Gleichungen für die Coefficienten der Fundamentalsubstitutionen. 
70 Otto Biermann: Über die Darstellung der Fuchs'schen etc.

Jetzt hat man die Bedingungen, unter welchen $F(z)$ eine Fuchs'sche Function wird, auf die nothwendigen und hinreichenden zuirückzufïhren. Wenn man die Fundamentalrelationen in allgemeiner Form niederschreibt, ist der Einblick in den Zusammenhang der Substitutionscoefficienten, und andererseits der willkürlichen Größen $C_{\mu}, e_{o}^{(x)}, c_{o}^{(\lambda)}$ nicht leicht; ich habe ihn noch nicht gewonnen und halte es für das beste, die Art und Form der verlangten Bedingungen zunächst an Beispielen zu suchen, damit man das Ziel deutlicher sehe. Doch auf Beispiele gehe ich an dieser Stelle nicht ein.

Nachträgliche Bemerkung. Die im 33. Bd. der math. Annalen enthaltene Abhandlung von Herrn Stahl über die Productdarstellung eindeutiger linearperiodischer Functionen wurde mir erst nach Beendigung meiner Arbeit bekannt, und leider fand ich bisher nicht die Zeit, jene Abhandlung zu verwerten. 